\title{
Obesity in the new millennium
}

\section{J. M. Friedman}

The Rockefeller University, Box 305, 1230 York Avenue, New York, New York 10021-6399, USA

Obesity has increased at an alarming rate in recent years and is now a worldwide public health problem. In addition to suffering poor health and an increased risk of illnesses such as hypertension and heart disease, obese people are often stigmatized socially. But major advances have now been made in identifying the components of the homeostatic system that regulates body weight, including several of the genes responsible for animal and human obesity. A key element of the physiological system is the hormone leptin, which acts on nerve cells in the brain (and elsewhere) to regulate food intake and body weight. The identification of additional molecules that comprise this homeostatic system will provide further insights into the molecular basis of obesity, and possibilities for new treatments.

W e live in an era in which advances in medical research reverberate almost instantly through society and culture. This statement is well illustrated by the visibility of recent progress in obesity research. As described in this timely issue, substantial advances have been made towards identifying the components of a physiological system that regulates body weight. Research in this area is at the centre of several important medical and societal issues. First, obesity is a pressing, some consider it the most pressing, health problem in Western and developing countries (see review by Kopelman, pp. 635-643, and ref. 1). Second, family studies of obesity provide a general opportunity for exploring the respective roles of genes and environment in determining human characteristics (see review by Barsh et al., pp. 644-651, and ref. 2). Third, research in this area has implications for the ways in which alterations of nutritional state affect the function of other organ systems $^{3}$. Finally, obesity research intersects with considerations of the molecular basis of human behaviour and even the nature of free will (see review by Schwartz et al., pp. 661-671, and refs 3, 4).

\section{Dichotomous views on the causes of obesity}

Because eating is an activity in which we all partake, it is not surprising that almost everyone has an opinion about this subject. (To paraphrase Paul Krugman in a recent New York Times editorial, this establishes obesity as a political issue.) Two essentially polar explanations characterize most views on the causes of human obesity.

One view suggests that obesity is the result of a fundamental lack of discipline on the part of affected individuals. This view is undoubtedly advanced by the diet industry

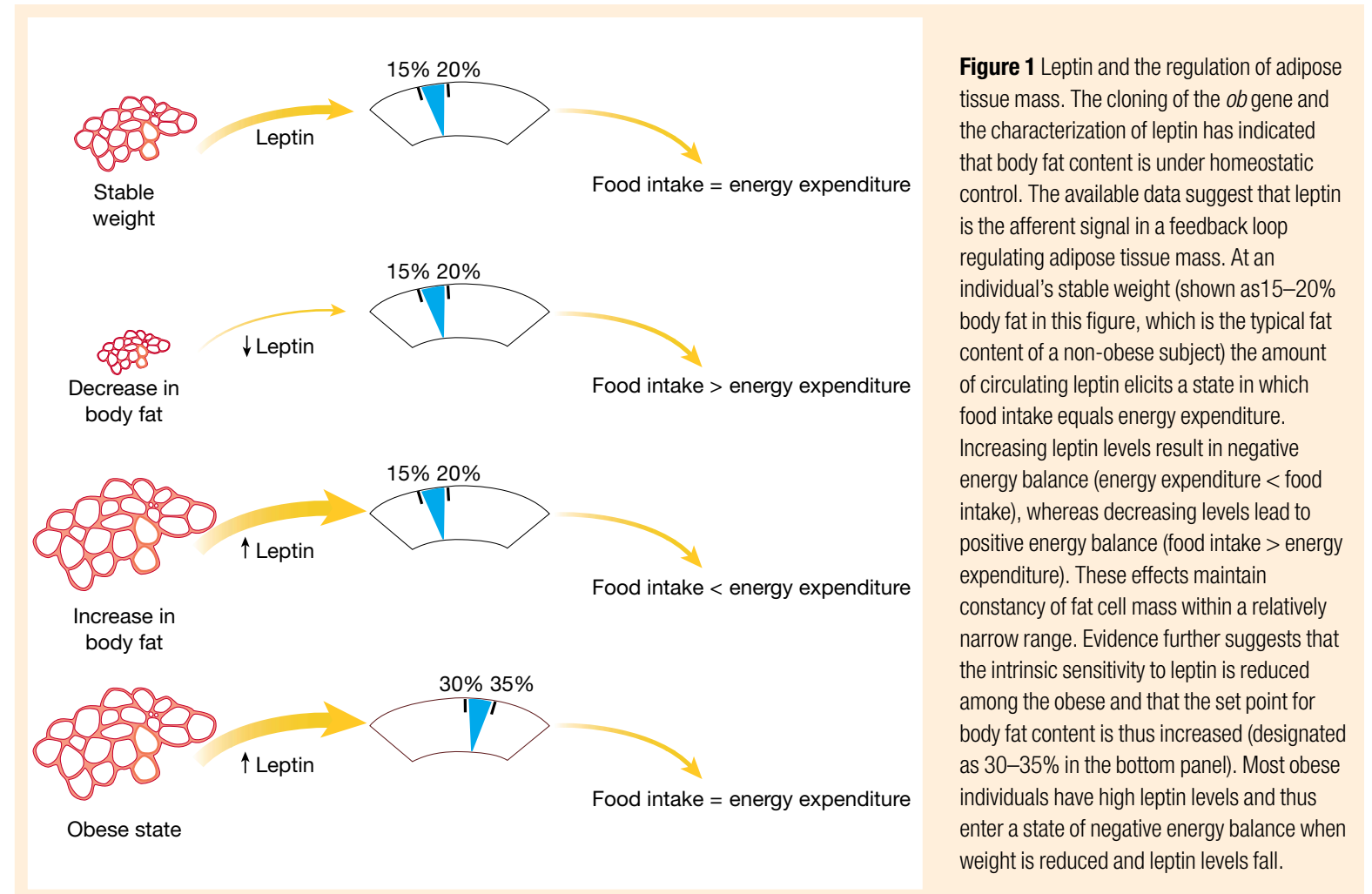




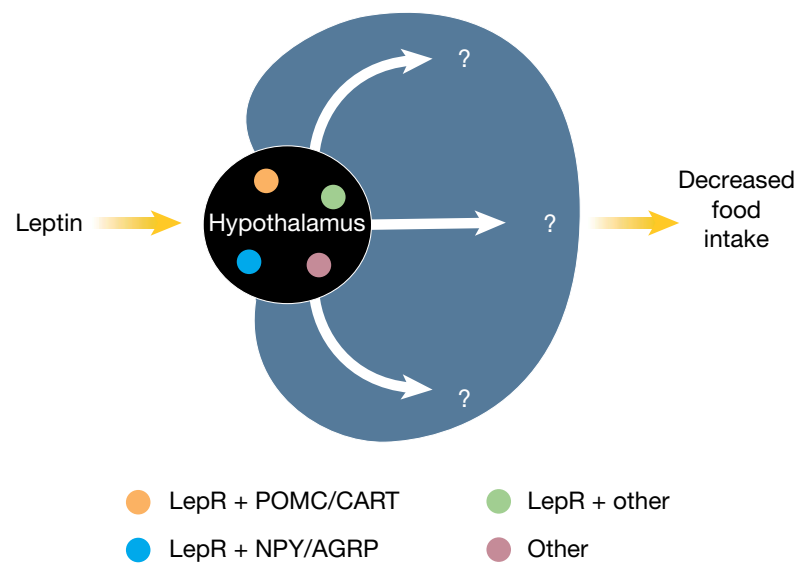

Figure 2 The neural circuit activated by leptin. In the arcuate nucleus of the hypothalamus the leptin receptor is expressed in at least two different classes of neurons. One class expresses NPY and AGRP, two neuropeptides that increase food intake. Another class expresses POMC, the precursor of $\alpha-\mathrm{MSH}$, and CART. Both CART and $\alpha$-MSH decrease food intake. Other leptin receptor-positive neurons do not express any of these molecules. The available evidence indicates that leptin suppresses the activity of NPY/AGRP neurons and stimulates the activity of POMC/CART neurons. Thus in the absence of leptin the NPY/AGRP neurons are maximally active and food intake is stimulated. In the presence of increased leptin levels the POMC/CART neurons are maximally active and food intake is reduced. When an individual is at their stable weight the activity of these pathways is balanced. The neural mechanisms by which these neurons ultimately change food intake are not known, as represented by the question marks. which has a financial interest (in aggregate greater than US $\$ 50$ billion each year) in promoting the notion that the only thing separating an individual from his or her 'dream' physique is the implementation of a few simple nostrums (provided by the company or author for a modest fee). Although it is true that reducing weight does improve the health of obese and overweight individuals, such remedies fail in the vast majority of cases ${ }^{5}$. Thus more than $90 \%$ of individuals who lose weight by dieting eventually return to their original weight.

The alternative view suggests that body weight (or more precisely, the amount of body fat) is physiologically controlled and that deviations in weight in either direction elicit a potent counter-response that resists that change ${ }^{6}$. Implicit in this view is the notion that biological factors determine each individual's body mass, be they lean or obese, and that this state is then defended. The effectiveness of this homeostatic system can be illustrated by a few simple calculations. Over the course of a decade, the weight of an average adult tends to increase slightly. Approximately 10 million kilocalories are consumed over this time. To account for the modest change of weight that is generally observed (assuming the excess weight is deposited as adipose tissue), food intake must precisely match energy output within $0.17 \%$ per decade ${ }^{7}$. This extraordinary level of precision has suggested that a robust biological system balances energy input (food intake) and energy expenditure. In recent years, this hypothesis has received substantial experimental support. These data are artfully reviewed in this issue and can be summarized as follows.

\section{A threat to life expectancy}

Obesity is formally defined as a significant increase above ideal weight, ideal weight being defined as that which maximizes life expectancy. Actuarial tables indicate that life expectancy is reduced when body-mass index (BMI; defined as mass in kilograms divided by the square of the height in metres), an indicator of adiposity or fatness, is significantly increased above the ideal level (see review by Kopelman, pp. 635-643, and refs 8, 9). This definition formally qualifies $20 \%$ of the US population, and a slightly lower percentage of the European population, as obese. There has also been an alarming increase in adolescent obesity in recent years ${ }^{10}$. Thus obesity is associated with a significant increase in morbidity and mortality and is a major public health problem. For reasons that are not fully known, obesity is associated with an increased risk of hypertension, heart disease, diabetes and cancer (see review by Kopelman, pp. 635-643). Even modest weight loss ameliorates these associated conditions.

In addition to the prospect of diminished health, obese people are often stigmatized both socially and in the workplace. Although the premium on leanness has become especially prominent in latetwentieth-century Western societies (at least among the affluent), this view is very dependent on the cultural context. In many cultures, obesity is considered to be a sign of affluence and prestige, particularly among those cultures where food is less available. In modern times, however, intense pressure to be thin is felt by most individuals, lean and obese. Despite this, obesity affects a significant and increasing number of individuals ${ }^{8}$.

Thus the critical question is, within a relatively homogenous environment, why are some individuals lean and others obese? The answer to this question has been informed by the identification of a number of genes that are responsible for animal and human obesity. Twin studies, analyses of familial aggregation and adoption studies all indicate that obesity is largely the result of genetic factors (see review by Barsh et al., pp. 644-651, and ref. 2). Indeed, the heritability of obesity is roughly equivalent to that of height and exceeds that of many disorders that are generally considered to have a genetic basis. The identity of several of these genes is now known and in these instances the evidence that obesity is not simply a personal failing is overwhelming.

\section{Leptin and body-weight regulation}

In general, obesity genes encode the molecular components of the physiological system that regulates body weight. A key element of this system is the hormone leptin ${ }^{3}$. Leptin is produced by fat tissue and reports nutritional information to key regulatory centres in a brain region known as the hypothalamus (Fig. 1). A decrease in body fat leads to a decreased level of this hormone, which in turn stimulates food intake. In addition, decreased leptin levels activate a hormonal response that is characteristic of the starved state ${ }^{11}$. Increased body fat is associated with increased levels of leptin, which act to reduce food intake. By such a mechanism, weight is maintained within a relatively narrow range. As would be predicted, mutations that result in leptin deficiency are associated with massive obesity in humans as well as rodents ${ }^{12,13}$. Leptin can also affect energy expenditure, which, in other contexts, is regulated independently of food intake (see review by Lowell and Spiegelman, pp. 652-660, and ref. 14). Changes in leptin concentration have effects on many other organ systems, including reproduction, the immune system and bone formation, which indicates that leptin is an important means by which changes in nutritional state affect physiology ${ }^{3}$.

Why then are some individuals obese and others not? It seems that the intrinsic sensitivity to leptin is variable and that, in general, obese individuals are leptin-resistant ${ }^{3,14,15}$. A smaller subset of individuals seems to produce too little leptin. In principle, genetic, environmental and even psychological factors could influence leptin sensitivity or leptin production. The molecular basis for leptin resistance has been explained in some instances. Leptin acts on nerve cells in the brain and modulates their function (Fig. 2). Several key molecules in this neural network are brain peptides known as neuropeptide Y (NPY) 
and agouti-related protein (AGRP), which stimulate food intake, and $\alpha$-melanocyte-stimulating hormone $(\alpha-\mathrm{MSH})$ and cocaine- and amphetamine-regulated transcript (CART), which decrease food intake (see review by Schwartz et al., pp. 661-671, and refs 2, 16, 17). These neural circuits also regulate energy expenditure by means of effects on several key molecules that have recentlybeen identified (see review by Lowell and Spiegelman, pp. 652-660). These effectors include uncoupling proteins and peroxisome proliferator-activated receptor- $\gamma$ (PPAR- $\gamma$ ) co-activator-1 (PGC-1), a key regulator of the genes that control thermogenesis ${ }^{18}$. Genetic evidence indicates that leptin regulates energy balance by modulating the balance among the aforementioned (and other) neuropeptides ${ }^{4}$. Mutations in proopiomelanocortin (POMC), the precursor of $\alpha-\mathrm{MSH}$, are associated with obesity ${ }^{19}$. In $\sim 3-5 \%$ of extremely obese individuals, mutations in an MSH receptor (MC4R) result in a defect in MSH signalling, which causes leptin resistance ${ }^{18,20,21}$. Mutation in the leptin receptor is also associated with extreme obesity ${ }^{22}$. In other cases it has been suggested that defective transport of leptin across the blood-brain barrier is the cause of leptin resistance and obesity ${ }^{3,23}$. Several other factors undoubtedly influence the function of this neural network, and the identification of additional molecules that comprise the neural system will shed more light on the molecular basis of obesity and leptin resistance.

\section{Impact of environmental factors}

There is plasticity of this system and such factors as diet, environment, age and perhaps exercise are also important in the pathogenesis of obesity (see review by Kopelman, pp. 635-643, and ref. 24). Thus the system that regulates weight sets a range of body weight in an individual and that range can be further influenced by other factors. For example, it is often noted that the incidence of obesity is rising dramatically in newly Westernized (so-called 'Coca Colanized') societies. In addition, the advent of a high-fat, Western diet is associated with a staggering increase in body weight among a number of native populations ${ }^{24,25}$. It is worth noting that a similar trend has also been observed for height in the twentieth century. The average US Civil War soldier was 5 feet 4 inches $(1.63 \mathrm{~m})$ tall, yet most people accept that biological factors contribute to differences in stature. The mechanisms by which environmental factors modulate the physiological system that controls weight are poorly understood, but in time they should prove tractable. Environmental factors have been shown to affect leptin sensitivity, as a high-fat diet leads to leptin resistance, although the basis for this is poorly understood ${ }^{3}$.

What then determines when we eat and how much we eat? Feeding behaviour is complex and is dependent on many factors, including olfactory, visual, emotional and higher cognitive inputs as well as leptin and several other nutritional signals. As the decision of whether or not to eat is the result of neurochemical events in the brain, and not metaphysical, there must be integratory centre(s) that balance all of these inputs. A fuller understanding of these neural events is likely to reveal the mechanisms by which complex behavioural decisions are made, not only for eating, but also perhaps for other complex behaviours.
Tremendous scientific opportunities abound. Recent insights into the molecular mechanisms that regulate weight have already led to numerous possibilities for new treatments and this trend will undoubtedly accelerate (see review by Bray and Tartaglia, pp. 672-677). But for the moment there is no panacea. So the final message is this...weight loss and exercise improve health and should be encouraged. However, a robust biological system makes it exceedingly difficult for most individuals to maintain weight loss for an extended period of time. This fact has deep implications for our perception of obesity and the obese.

\footnotetext{
1. Calle, E., Thun, M., Petrelli, J., Rodriquez, C. \& Heath, C. Body-mass index and mortality in a prospective cohort of U.S. adults. N. Engl. J. Med. 341, 1097-1105 (1999).

2. Stunkard, A. J., Harris, J. R., Pedersen, N. L. \& McClearn, G. E. The body-mass index of twins who have been reared apart. N. Engl. J. Med. 322, 1483-1487 (1990).
}

3. Friedman, J. M. \& Halaas, J. L. Leptin and the regulation of body weight in mammals. Nature 395 , 763-770 (1998).

4. Elias, C. et al. Leptin differentially regulates NPY and POMC neurons projecting to the lateral hypothalamic area. Neuron 23, 775-786 (1999).

5. Wadden, T. A. Treatment of obesity by moderate and severe caloric restriction. Results of clinical research trials. Ann. Intern. Med. 119, 688-693 (1993).

6. Kennedy, G. C. The role of depot fat in the hypothalamic control of food intake in the rat. Proc. R. Soc. Lond. B 140, 578-592 (1953).

7. Weigle, D. S. Appetite and the regulation of body composition. FASEB J. 8, 302-310 (1994),

8. Kuczmarski, R., Flegal, K., Campbell, S. \& Johnson, C. Increasing prevalence of overweight among US adults. J. Am. Med. Assoc. 272, 205-211 (1994).

9. Manson, J. E. et al. Body weight and mortality among women. N. Engl. J. Med. 333, 677-685 (1995).

10. Troiano, R. \& Flegal, K. Overweight children and adolescents: description, epidemiology, and demographics. Pediatrics 101, 497-504 (1998).

11. Ahima, R. S. et al. Role of leptin in the neuroendocrine response to fasting. Nature 382, 250-252 (1996).

12.Zhang, Y. et al. Positional cloning of the mouse obese gene and its human homologue. Nature 372, 425-432 (1994).

13. Montague, C. T. Congenital leptin deficiency is associated with severe early-onset obesity in humans. Nature 387, 903-908 (1997).

14. Pelleymounter, M. A. et al. Effects of the obese gene product on body weight regulation in $o b / o b$ mice. Science 269, 540-543 (1995).

15. Maffei, M. et al. Leptin levels in human and rodent: measurement of plasma leptin and $o b$ RNA in obese and weight-reduced subjects. Nature Med. 1, 1155-1161 (1995).

16. Erickson, J. C., Hollopeter, G. \& Palmiter, R. D. Attenuation of the obesity syndrome of ob/ob mice by the loss of neuropeptide Y. Science 274, 1704-1707 (1996).

17. Fan, W., Boston, B. A., Kesterson, R. A., Hruby, V. J. \& Cone, R. D. Role of melanocortinergic neuron in feeding and the agouti obesity syndrome. Nature 385, 165-168 (1997).

18. Puigserver, P. et al. A cold-inducible coactivator of nuclear receptors linked to adaptive thermogenesis. Cell 92, 829-839 (1998).

19. Krude, H. et al. Severe early-onset obesity, adrenal insufficiency and red hair pigmentation caused by POMC mutations in humans. Nature Genet. 19, 155 (1998).

20. Yeo, G. et al. A frameshift mutation in MC4R associated with dominantly inherited human obesity. Nature Genet. 20, 111-112 (1998).

21.Vaisse, C., Clement, K., Guy-Grand, B. \& Froguel, P. A frameshift mutation in human MC4R is associated with a dominant form of obesity. Nature Genet. 20, 113-114 (1998).

22. Clement, K. et al. A mutation in the human leptin receptor gene causes obesity and pituitary dysfunction. Nature 392, 398-401 (1998).

23. Schwartz, M. W., Peskind, E, Raskind, M. Boyko, E. J. \& Porte, D. Jr Cerebrospinal fluid leptin levels: relationship to plasma levels and to adiposity in humans. Nature Med. 2, 589-593 (1996).

24. James, W. in The Origins and Consequences of Obesity (eds Chadwick, D. \& Cardew, G.) 1-16 (Wiley, Chichester, 1996).

25. Ravussin, E. Metabolic differences and the development of obesity. Metabolism 9, 12-14 (1995).

Acknowledgements

I thank S. Korres for assistance in preparing this manuscript and J. Breslow, J. Darnell, S. Friedman, A. Soukas, M. Stoffel and E. Rupple-Shell Stoffel for critical and thoughtful comments. 